\title{
Targeted sequencing reveals distinct pathogenic variants in Chinese patients with lung adenocarcinoma brain metastases
}

\author{
YANCHUN MA $^{1 *}, \mathrm{KUN} \mathrm{CHEN}^{1 *}, \mathrm{ZHENHUA} \mathrm{YANG}^{2}$ and MING GUAN ${ }^{1,3}$ \\ ${ }^{1}$ Department of Laboratory Medicine, Huashan Hospital North, Fudan University, Shanghai 201907; \\ ${ }^{2}$ Clinical Laboratory, Baoshan District Hospital of Integrated Traditional Chinese and Western Medicine, Shanghai 201999; \\ ${ }^{3}$ Central Laboratory, Huashan Hospital, Shanghai Medical School, Fudan University, Shanghai 200000, P.R. China
}

Received March 12, 2017; Accepted December 6, 2017

DOI: $10.3892 / \mathrm{ol} .2018 .7859$

\begin{abstract}
Lung cancer is the most common type of malignancy to metastasize to the brain, with the median survival time of patients being 6-11 months. In the present study, the aim was to compare the actionable gene mutation profiles of primary lung adenocarcinoma (LC) samples and LC brain metastasis (LCBM) samples through targeted sequencing. Next generation sequencing (NGS) of 13 formalin-fixed, paraffin-embedded LC samples and 15 LCBM samples was performed using a customized OncoAim $^{\mathrm{TM}}$ cancer panel and OncoAim ${ }^{\mathrm{TM}}$ RNA fusion panel on the MiSeq platform. The OncoAim ${ }^{\mathrm{TM}}$ cancer panel pipeline and OncoAim ${ }^{\mathrm{TM}}$ RNA fusion panel pipeline were used for bioinformatic analysis. Together, 43 variants were observed in 7 genes from the 28 cancer samples. The mutated genes of LCBM were tumor protein (TP)53, epidermal growth factor receptor (EGFR), catenin $\beta 1$, phosphatidylinositol-4,5-bisphosphate 3 -kinase catalytic subunit $\alpha$, mothers against decapentaplegic homolog 4, Kirsten rat sarcoma viral oncogene homolog (KRAS) and proto-oncogene B-Raf, which were exhibited in 10/15 (66.7\%), 6/15 (40.0\%), 3/15 (20.0\%), 2/15 (13.3\%), $2 / 15$ (13.3\%), 1/15 (6.7\%) and $1 / 15(6.7 \%)$ of samples, respectively. The mutated genes of LC were TP53, EGFR and KRAS, which were exhibited in 11/13 (84.6\%), $5 / 13(38.5 \%)$ and $2 / 13(18.2 \%)$ of samples, respectively. echinoderm microtubule associated protein like 4-anaplastic
\end{abstract}

Correspondence to: Professor Zhenhua Yang, Clinical Laboratory, Baoshan District Hospital of Integrated Traditional Chinese and Western Medicine, 181 Youyi Road, Baoshan, Shanghai 201999, P.R. China

E-mail: shbsyzh@126.com

Professor Ming Guan, Department of Laboratory Medicine, Huashan Hospital North, Fudan University, 108 Luxiang Road, Baoshan, Shanghai 201907, P.R. China

E-mail: guanming88@yahoo.com

*Contributed equally

Key words: gene mutations, brain metastases, lung adenocarcinoma, next-generation sequencing, targeted therapy lymphoma kinase rearrangements were present in $1 \mathrm{LCBM}$ sample. For 2 LC samples and 1 LCBM sample, no genetic alterations were observed. The NGS data also revealed a novel 4-codon deletion of TP53 (p.V166_H169del) and a novel TP53 splice site mutation (7577157-63del TACTCAG). Further potentially actionable mutations were detected in LCBM, indicating a high degree of genetic heterogeneity between the LC and LCBM samples that were analyzed. The present study demonstrated that NGS provides an improved approach for the discovery of potentially actionable mutations and the understanding of the mechanisms underlying tumor progression and evolution.

\section{Introduction}

Brain metastases (BM), particularly those from non-small cell lung cancer (NSCLC), constitute an increasing proportion of the global cancer burden. It is estimated that $50 \%$ of all patients with BM will experience a primary lung malignancy (1). In patients with central nervous system (CNS) metastases, the administration of standard chemotherapies or targeted agents is limited due to low CNS penetration of antineoplastic agents across the blood-brain barrier (2).

However, in the last decade, several biological agents targeting specific molecular alterations, including gefitinib or erlotinib targeting epidermal growth factor receptor (EGFR) mutations and crizotinib targeting anaplastic lymphoma kinase (ALK) translocations, have been approved for clinical use and used in personalized treatment regimes (3-7). Selected molecular-targeted therapy for BM of lung cancer has been notably effective, demonstrated by response rates, progression-free survival and quality of life in small studies and case reports (8-11); however, there are limited data available regarding the differential genetic aberrations between lung adenocarcinoma BM (LCBM) and primary lung cancer (LC) (12).

Next-generation sequencing (NGS) technologies provide an unprecedented opportunity to identify the clinically actionable mutations relevant to personalized cancer care, and are increasingly used for the mutational analysis of tumors for clinical and research applications (13-15). Targeted NGS permits the concurrent deep sequencing of hundreds of cancer genes, and has emerged as a sensitive 
and efficient tool to detect complex and heterogeneous gene mutations (16-18).

In the present study, targeted NGS was conducted to detect potentially actionable genetic aberrations in LCBM as well as primary LC, to provide target gene mutation profiles and an improved understanding of the genetic mechanism underlying lung cancer metastasis. The aim of the present study was to identify novel and potentially beneficial therapeutic targets for patients with BM.

\section{Materials and methods}

Patients. A total of 28 formalin-fixed, paraffin-embedded (FFPE) tumor samples, including 13 cases of LC and 15 cases of LCBM, were retrospectively collected from the archives of the Pathology Laboratory in Huashan Hospital North in Fudan University (Shanghai, China) between January 2013 and December 2015. There were 10 unstained FFPE tissue sections $(10 \mu \mathrm{m})$ that were cut for DNA and RNA extraction. The pathologist identified the lesional area and the percentage tumor cell content (minimum of 50\%) for each case, which were the areas microdissected for extraction. Assessment of $\mathrm{BM}$ was concluded using all of the radiology reports, which contained brain imaging or pathology.

DNA and RNA extraction from FFPE samples. Genomic DNA was extracted from FFPE slices of solid tumor using the QiagenQIAamp DNA FFPE kit (Qiagen, Inc., Valencia, CA, USA), according to the manufacturer's protocol. DNA was eluted with $40 \mu$ l elution buffer (10 mM Tris-Cl, $\mathrm{pH} 8.3$; $0.1 \mathrm{mM}$ EDTA; $0.04 \% \mathrm{NaN}_{3}$ ). The concentration was measured using the Qubit HS kit (Thermo Fisher Scientific, Inc., Waltham, MA, USA). Total cellular RNA was extracted using the QiagenRNeasy FFPE kit, according to the manufacturer's protocol with minor modifications as specified below (Qiagen, Inc.). A total of $1 \mathrm{ml}$ xylene was added to the $1.5 \mathrm{ml}$ tube containing the FFPE tissue. The tube was vortexed vigorously for $10 \mathrm{sec}$ and centrifuged at 21,130 x g for $2 \mathrm{~min}$ at $25^{\circ} \mathrm{C}$. The supernatant was carefully removed using a pipette. Then $1 \mathrm{ml}$ ethanol (96-100\%) was added into the tube, followed by vigorous vortexing followed by centrifugation at $21,130 \mathrm{x} \mathrm{g}$ for $2 \mathrm{~min}$ at $25^{\circ} \mathrm{C}$. The above step was repeated once. Then, the ethanol was carefully removed. The tube was kept open at room temperature $\left(15-25^{\circ} \mathrm{C}\right)$ for 10 min until all residual ethanol evaporated. Then, the manufacturer's protocol (Qiagen, Inc.) was followed, adding $150 \mu \mathrm{l}$ Buffer PKD and following the remaining steps in order to obtain RNA. The RNA concentration was measured with the Qubit ${ }^{\circledR}$ RNA kit (Thermo Fisher Scientific, Inc.), and RNA integrity was evaluated using RNA gel electrophoresis (1\% agarose gel in $0.5 \%$ TBE buffer, running for $35 \mathrm{~min}$ at a $120 \mathrm{v}$ voltage; with a ratio of $28 \mathrm{~S}$ rRNA to $18 \mathrm{~S}$ rRNA of $\sim 2: 1$ ). Reverse transcription of RNA to cDNA was conducted using the SuperScript ${ }^{\mathrm{TM}}$ VILO $^{\text {TM }}$ cDNA synthesis kit (Thermo Fisher Scientific, Inc.), and was followed by library construction.

Sequencing library construction. The OncoAim ${ }^{\mathrm{TM}}$ cancer panel (Singlera Genomics, Inc., Shanghai, China) was used with the recommended DNA input (20 ng for FFPE samples) to generate sequencing libraries compatible with MiSeq system
Dx (DX-410-1001; llumina, Inc., San Diego, CA, USA). The panel included 400 amplicons targeting cancer hotspots, the specific genetic loci of which were depicted on the website of the Singlera Genomics, Inc. (Shanghai, China; http://www. singlera.com.cn/product/index.aspx?pid=1). The targeted regions were amplified using the customized OncoAim ${ }^{\mathrm{TM}}$ cancer panel followed by the addition of P5/P7 dual barcode adapters (Illumina, Inc.).

In addition, $20 \mathrm{ng}$ reverse transcribed cDNA was amplified with the OncoAim ${ }^{\mathrm{TM}}$ RNA fusion panel (Singlera Genomics, Inc.) to generate sequencing libraries compatible with MiSeq. The panel included 170 amplicons targeting fusion events for ALK, Ret proto-oncogene, proto-oncogene c-Ros1 and neurotrophic receptor tyrosine kinase, along with five genes (hydroxymethulbilane synthase, integrin subunit $\beta$, lamin A/C, MYC proto-oncogene, bHLH transcription factor and TATA-box binding protein) as internal expression controls. Fusion variants, if present, were enriched by the customized OncoAim $^{\mathrm{TM}}$ RNA fusion panel followed by a P5/P7 addition (Illumina, Inc.).

LabChip ${ }^{\circledR}$ GX Touch (PerkinElmer, Inc., Waltham, MA, USA) was used to confirm successful library amplifications to a desired length ( $220 \mathrm{bp}$ ), and library concentration was assessed using the Qubit ${ }^{\mathrm{TM}}$ HS kit (Thermo Fisher Scientific, Inc.). No detectable DNA in the library prepared from non-template control (negative control) was used to indicate that no DNA contamination was introduced during library preparation. There were 12-16 barcoded libraries that were pooled. The ratios were confirmed and quantified by qPCR using KAPA Library Quantification kit (Kapa Biosystems, Inc., Wilmington, MA, USA) following the manufacturer's protocol, using the Bio-Rad CFX96 qPCR system (Bio-Rad Laboratories, Inc., Hercules, CA, USA). The KAPA Library Quantification kit uses the novel KAPA SYBR FAST DNA Polymerase, engineered through a process of directed evolution for high-performance SYBR Green I-based qPCR (Kapa Biosystems, Inc., Wilmington, MA, USA). The qPCR primers are 5'-AATGATACGGCGACCACCGA-3' and 5'-CAAGCAGAAGACGGCATACGA-3'. The PCR cycling conditions were: initial denaturation: $95^{\circ} \mathrm{C}$ for $5 \mathrm{~min}$; cycling: 35 cycles of $95^{\circ} \mathrm{C}$ for $30 \mathrm{sec}$ and $60^{\circ} \mathrm{C}$ for $45 \mathrm{sec}$. The library concentration was calculated against the standard curve generated from 6 standards using qPCR software on the Bio-Rad CFX96 qPCR system (CFX Maestro software 12004110; Bio-Rad Laboratiories, Inc.).

Data processing. A bioinformatic analysis pipeline was created using a corresponding OncoAim ${ }^{\mathrm{TM}}$ cancer panel pipeline $\left(\right.$ OncoAim ${ }^{\mathrm{TM}}$ version 7.2) and OncoAim ${ }^{\mathrm{TM}}$ RNA fusion panel pipeline (OncoAim ${ }^{\mathrm{TM}}$ RNA version 3.1). Briefly, raw reads (fastq files) were quality-filtered with FastQC version 0.9.5 (Babraham Bioinformatics, Cambridge, UK) and base quality score recalibration was performed. Clean reads were assembled and aligned against the reference genome hg19, or the targeted fusion reference to detect sequence/structure variations. The Burrow-Wheeler Aligner algorithm (https://github.com/lh3/bwa; version 0.7.12-r1039; Dec 2015) was implemented into the procedure for alignment, using default parameters. Insertions and deletions in sequence alignment files were left-aligned, and local realignment was 
conducted with the Genome Analysis Toolkit (https://github. com/broadgsa/gatk; Broad Institute, Cambridge, MA, USA) and Freebayes (https://github.com/ekg/freebayes; version 0.9.21-26-gbfd9832). Unique reads derived from the GATK were used for variant calling. All samples were processed in parallel, and each sample had split individual variant files. The median coverage/locus was 500-1,000 to ensure confident variant calling. The minimum confidence threshold for variant and insertion/deletion (indel) calling was set to $0.05(5 \%)$. Single nucleotide changes (SNC) and indels were separately evaluated. Fusion transcripts were normalized to total mapped reads to calculate the CP100 K (counts/100,000 mapped reads), for a fair comparison across different libraries. Variation annotation, effect prediction and clinical practice guidance, including The National Comprehensive Cancer Network (www.nccn.org), were integrated into the OncoAim ${ }^{\mathrm{TM}}$ cancer panel pipeline through vcf files. Additionally, descriptive statistics and data visualization were performed using Microsoft Excel 2010 (Microsoft Corporation, Redmond, WA, USA) and R packages (version 3.4.1; http://cran.r-project. org/src/base/R-3/R-3.4.1.tar.gz). The Integrative Genomics Viewer (version 2.3.94, Broad Institute) was used for visualization and confirmation of specific SNC/indel loci.

Confirmation of mutations using Sanger sequencing. A total of 2 samples with novel tumor protein (TP)53 mutations were selected from targeted NGS for validation testing using primer-specific PCR and Sanger sequencing. Sanger sequencing was performed using a DNA direct sequencing company (Sangon Biotech Co., Ltd., Shanghai, China). TP53-p.V166_H169del primer: forward, 5'-GTTTCTTTG CTGCCGTCTT C-3' and reverse, 5'-CTTAACCCCTCCTCC CAGAG-3'. TP53-7577157-63del TACTCAG primer: Forward, 5'-CTTGGGCCTGTGTTATCTCC-3' and reverse, 5'-TAA CTGCACCCTTGGTCTCC- 3'.

Statistical analysis. Descriptive statistics were calculated with Microsoft Excel 2010 (Microsoft Corp, Redmond, Wash). The data were described as the number of mutations. All statistical tests were performed using IBM SPSS Statistics version 19 (IBM Corp., Armonk, NY, USA). The $\chi^{2}$ test or Fisher's exact test was used to calculate the P-values for the detected number of mutations or the frequency of mutated genes between LC and LCBM samples. $\mathrm{P}<0.05$ was considered to indicate a statistically significant difference.

\section{Results}

Patient characteristics. Characteristics of the 13 patients with LC and 15 patients with LCBM are presented in Table I. The median age of patients diagnosed with LC and LCBM was 61 years (range, 50-74 years) and 55 years (range, 35-65 years), respectively. Overall, $57 \%$ of patients (16 cases) were male, and the majority of patients (24 cases) did not have a history of smoking. The majority of patients $(89.3 \%, 25 / 28)$ were diagnosed in advanced (tumor-node-metastasis III and IV) stages (19).

NGS. A total of 28 samples were successfully sequenced. Table II presents the results of NGS of 28 samples with the
Table I. Baseline characteristics of all patients $(n=28)$.

\begin{tabular}{lcc}
\hline Characteristics & LC $(\mathrm{n}=13)$ & LCBM $(\mathrm{n}=15)$ \\
\hline Age, years & & 55 \\
Median & 61 & $35-65$ \\
Range & $50-74$ & \\
Sex & & 7 \\
Male & 9 & 8 \\
Female & 4 & \\
Smoking history & & 15 \\
Never smoked & 9 & 0 \\
Have smoked & 4 & LCBM \\
Pathological diagnosis & LC & \\
TNM stage & & 0 \\
Ia-IIb & 3 & 0 \\
IIIa-IIIb & 10 & 15 \\
IV & & Yes \\
BM & No & \\
\hline
\end{tabular}

LC, lung adenocarcinoma; LCBM, lung adenocarcinoma brain metastasis; TNM, tumor node metastasis; BM, brain metastasis.

OncoAim ${ }^{\mathrm{TM}}$ cancer panel and the presence of each sample gene mutation can be observed in Fig. 1. The mutated genes in these 28 samples were TP53, EGFR, Kirsten rat sarcoma viral oncogene homolog (KRAS), catenin $\beta 1$ (CTNNB1), phosphatidylinositol-4,5-bisphosphate 3-kinase catalytic subunit $\alpha$ (PIK3CA), mothers against decapentaplegic homolog 4 (SMAD4) and proto-oncogene B-Raf (BRAF). The number of mutant genes are summarized in Fig. 2A, and were identified in $21 / 28(75.0 \%), 11 / 28$ (39.3\%), $3 / 28(10.7 \%), 3 / 28(10.7 \%)$, $2 / 28(7.1 \%), 2 / 28(7.1 \%)$ and $1 / 28(3.6 \%)$ of samples, respectively. Overall, 43 variants in the 7 genes were observed. These 43 genomic variant aberrations included 36 base substitutions and 7 short deletions. A total of 5 samples $(5 / 28,18 \%)$ exhibited 3 potentially biologically significant variants, 8 samples $(8 / 28,29 \%)$ exhibited 2 variants, 11 samples $(11 / 28,39 \%)$ exhibited only a single variant, and 4 samples $(4 / 28,14 \%)$ exhibited no alterations (Fig. 2B). The average number of variants was $1.5 /$ tumor. The RNA fusion panel tests revealed only echinoderm microtubule associated protein like 4 [EML4 (13)]-ALK (20) in 1sample of LCBM (Fig. 3).

Comparison of gene mutations between LC and LCBM. The mutated genes in LCBM tissues were TP53, EGFR, CTNNB1, PIK3CA, SMAD4, KRAS and BRAF, which were identified in $10 / 15$ (66.7\%), 6/15 (40.0\%), 3/15 (20.0\%), 2/15 (13.3\%), $2 / 15(13.3 \%), 1 / 15(6.7 \%)$ and $1 / 15(6.7 \%)$ of samples, respectively. The mutated genes of LC were TP53, EGFR and KRAS, which were exhibited in 11/13 (84.6\%), 5/13 (38.5\%) and 2/13 $(18.2 \%)$ of samples, respectively. EML4-ALK rearrangement existed in $1 \mathrm{LCBM}$ sample. A total of $2 \mathrm{LC}$ samples and $1 \mathrm{LCBM}$ sample exhibited no genetic alterations (Fig. 4). No significant differences were identified in the number of mutations or the frequency of TP53, EGFR or KRAS between patients with LC $(n=13)$ and patients with LCBM $(n=15 ; P>0.05)$. 
Table II. Mutation profiles in 28 clinical samples.

\begin{tabular}{lccc}
\hline & LC & LCBM & Mutation \\
Mutant gene & $(n=13)$ & $(n=15)$ & frequency \\
\hline
\end{tabular}

\section{TP53}

$21 / 28$

p.T125T
p.P151A
p.V173L
p.R175H
p.C176F
p.E180G
p.D207G
p.Y234C
p.Y236Y
p.M237V
p.G245C
p.T253I
p.R267P
p.R273L
p.V166_H169del
7577157-63del TACTCAG

EGFR

Exon21

$$
\text { p.L858R }
$$

1

$$
1
$$

\section{1}

4

1

1

1

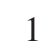

1

Exon 19

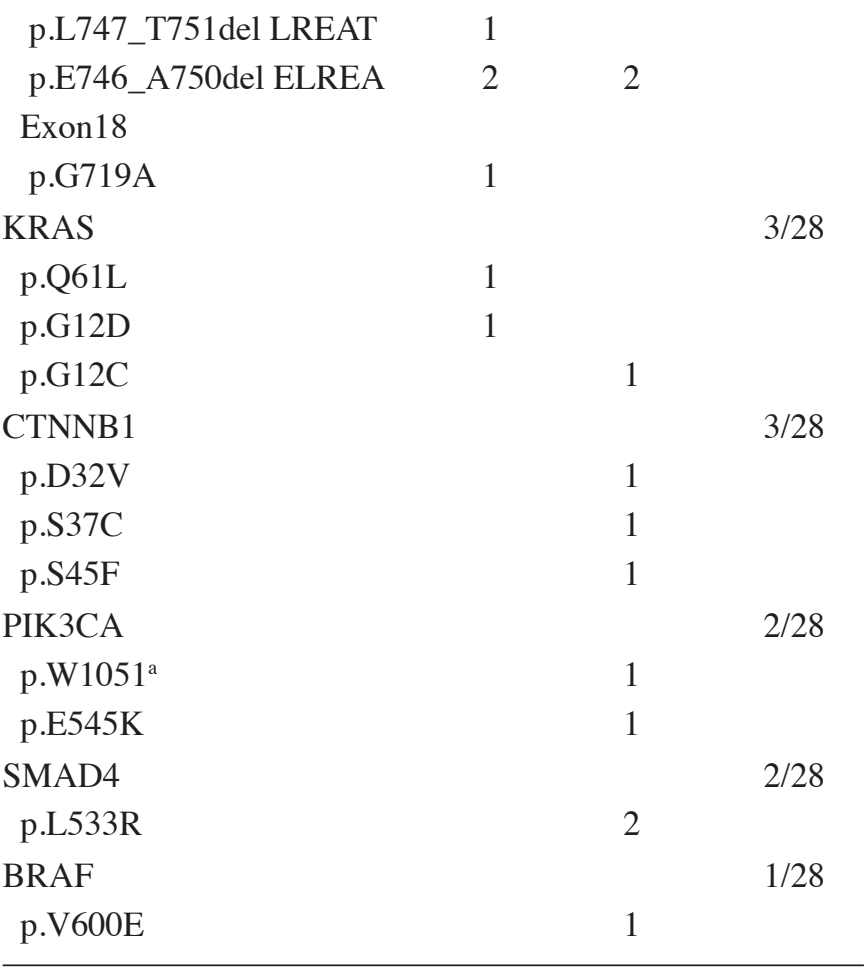

aTermination codon-the mutation causes the translation to be terminated prematurely. LC, lung adenocarcinoma; LCBM, lung adenocarcinoma brain metastasis; TP53, tumor protein 53; EGFR, epidermal growth factor receptor; KRAS, Kirsten rat sarcoma viral oncogene; CTNNB1, catenin $\beta-1$; PIK3CA, phosphatidylinositol4,5-bisphosphate 3-kinase; BRAF, proto-oncogene B-Raf; SMAD4, mothers against decapentaplegic homolog 4 .
Novel TP53 gene mutations in LCBM and mutation verification by Sanger sequencing. The LCBM-7 and LCBM-11 samples exhibited a novel TP53 mutation form p.V166_H169del and a new splice site mutation for 7577157-63del TACTCAG, respectively, located in the non-coding regions. Fig. 5A depicts the Sanger sequencing chromatograms of p.V166_H169del compared with the wild-type (Fig. 5B). Fig. 5C depicts the Sanger sequencing chromatograms of 7577157-63del TACTCAG compared with the wild-type (Fig. 5D).

\section{Discussion}

Lung cancer is the most common cause of cancer-associated mortality globally, and 80-85\% of these cases are NSCLC (20). In total, $10-50 \%$ of patients with NSCLC develop BM (21). Treatment options are palliative, and usually include a combination of steroids, radiation and surgery (22). Despite advances in cancer therapy, the median survival time for patients with lung cancer BM is only 6-11 months (23). The poor prognosis associated with disseminated disease that has metastasized to the brain is not addressed by existing therapies. Efforts to develop improved personalized therapies and prognostic biomarkers must be driven by the understanding of the mechanisms underlying this disease. In the present study, a genetic alteration profile of the BM was produced, as well as for primary LC, with the intention of identifying potential biomarkers associated with brain metastasis from lung cancer. Currently, limited numbers of biomarkers possess the potential to be used clinically for BM, however a large number of promising candidate biomarkers are being evaluated in current studies, and may soon become relevant for clinical practice (12).

The targeted NGS analysis of 15 tumor samples from patients with LCBM as well as 13 patients with primary LC revealed that $14 / 15(93.3 \%)$ patients exhibited $\geq 1$ somatic molecular activating mutation and 11/13 (84.6\%) had $\geq 1$ molecular aberration. The most frequent genomic alterations in these types of cancer were TP53 (11 for LC, 10 For LCBM), KRAS (1 for LC, 2 for LCBM) and EGFR ( 5 for LC, 6 for LCBM). Notably, mutations in CTNNB1, BRAF, SMAD4 and PIK3CA were identified only in LCBM, although their frequencies were low.

In the present study, $60.7 \%(17 / 28)$ of the lung cancer samples analyzed exhibited a TP53 mutation, which is higher than that reported in a number of studies from Western countries $(24,25)$. This discrepancy may be explained by relatively few early-stage samples being analyzed in the present cohort. Lung cancer tissues exhibiting EGFR mutations have demonstrated success in responding to EGFR tyrosine kinase inhibitors $(26,27)$. An equal distribution of EGFR mutations in exon $19(5 / 28)$ and the L858R point mutation in exon $21(5 / 28)$ were identified in the present study, followed by the p.G719A point mutation in exon $8(1 / 28)$.

KRAS mutations were observed in patients with LC in a previous study; KRAS and other driver mutations, including EGFR, human epidermal growth factor receptor 2 and BRAF, being mutually exclusive, as KRAS mutations may represent negative predictive biomarkers for tumor responses in patients with NSCLC treated with EGFR-TKIs (28). Whereas EGFR mutations were identified more frequently in never-smokers, 


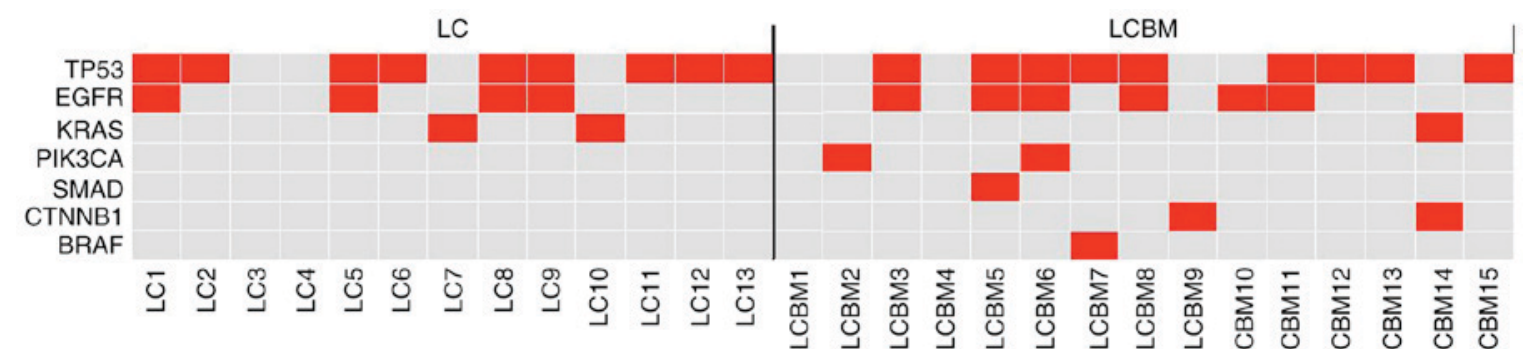

Figure 1. Heat map depicting the somatic mutations identified in each sample. Red indicates variants, whereas gray indicates no variants detected. The y-axis indicates the gene name and the $\mathrm{x}$-axis indicates the identification of the matched pairings, including 13 samples of LC and 15 samples of LCBM. LCBM, lung adenocarcinoma brain metastasis; TP53, tumor protein 53; EGFR, epithelial growth factor receptor; CTNNB1, catenin- $\beta 1$; KRAS, Kirsten rat sarcoma viral oncogene; PIK3CA, phosphatidylinositol-4,5-bisphosphate 3-kinase; BRAF, proto-oncogene B-Raf; SMAD4, mothers against decapentaplegic homolog 4.

A

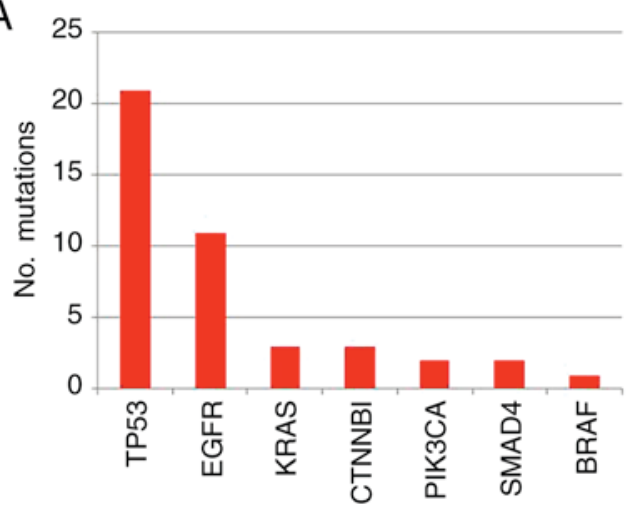

B

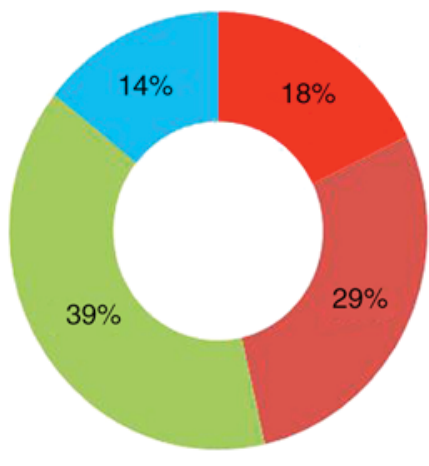

$5(18 \%)$ samples 3 variants

$8(29 \%)$ samples 2 variants

$11(39 \%)$ samples 1 variants

$4(14 \%)$ samples 0 variants

Figure 2. (A) Mutations identified in 28 samples and their distribution. (B) Distribution of samples according to variants identified. TP53, tumor protein 53; EGFR, epithelial growth factor receptor; CTNNB1, catenin- $\beta 1$; KRAS, Kirsten rat sarcoma viral oncogene; PIK3CA, phosphatidylinositol-4,5-bisphosphate 3-kinase gene; BRAF, proto-oncogene B-Raf; SMAD4, mothers against decapentaplegic homolog 4.

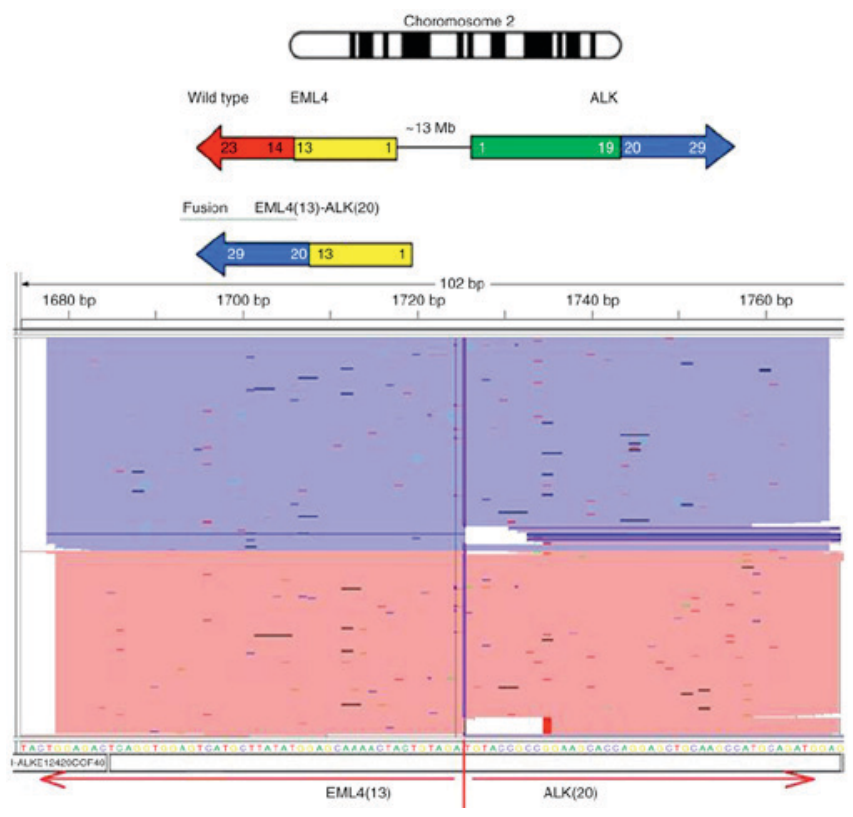

Figure 3. RNA fusion panel tests revealed only EML4(13)-ALK(20) in one sample of lung adenocarcinoma brain metastasis. EML4, echinoderm microtubule associated protein like 4; ALK, anaplastic lymphoma kinase. The blue bar represents the Cris, and the pink bar represents the Watson strand of DNA in the reference genome (hg19).

KRAS mutations were first identified among patients who reported greater tobacco use (29). KRAS mutations were

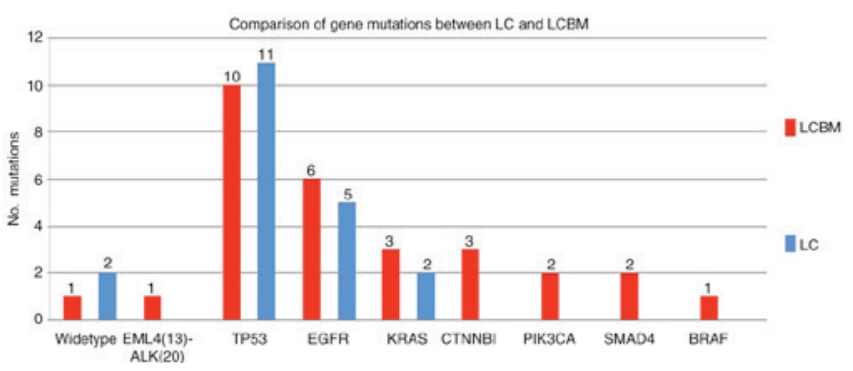

Figure 4. Variant frequency detected by next generation sequencing in 28 formalin-fixed, paraffin-embedded samples sub-grouped by LC and LCBM. CTNNB1, PI3KCA, SMAD4, BRAF and EML4-ALK mutations were present in the metastatic lesions in $3 / 15,2 / 15,2 / 15,1 / 15$ and 1/15 LCBM cases, but not in primary LC. LCBM, lung adenocarcinoma brain metastasis; LC, lung adenocarcinoma; TP53, tumor protein 53; EGFR, epithelial growth factor receptor; CTNNB1, catenin- $\beta 1$; KRAS, Kirsten rat sarcoma viral oncogene; PIK3CA, phosphatidylinositol-4,5-bisphosphate 3-kinase gene; BRAF, proto-oncogene B-Raf; EML4, echinoderm microtubule associated protein Like 4; ALK, anaplastic lymphoma kinase; SMAD4, mothers against decapentaplegic homolog 4 .

identified in $20-30 \%$ of patients with lung cancer in North American populations (30). The prevalence of KRAS mutations in the present study was less than that observed in the Caucasian group. Similarly, a recent study also identified KRAS mutations in $8.3 \%(113 / 1,368)$ of a patient cohort with LC (31). In the present study, BRAF and PIK3CA mutations were identified in a small proportion of patients with LCBM, however were not exhibited in patients with LC. However, these 

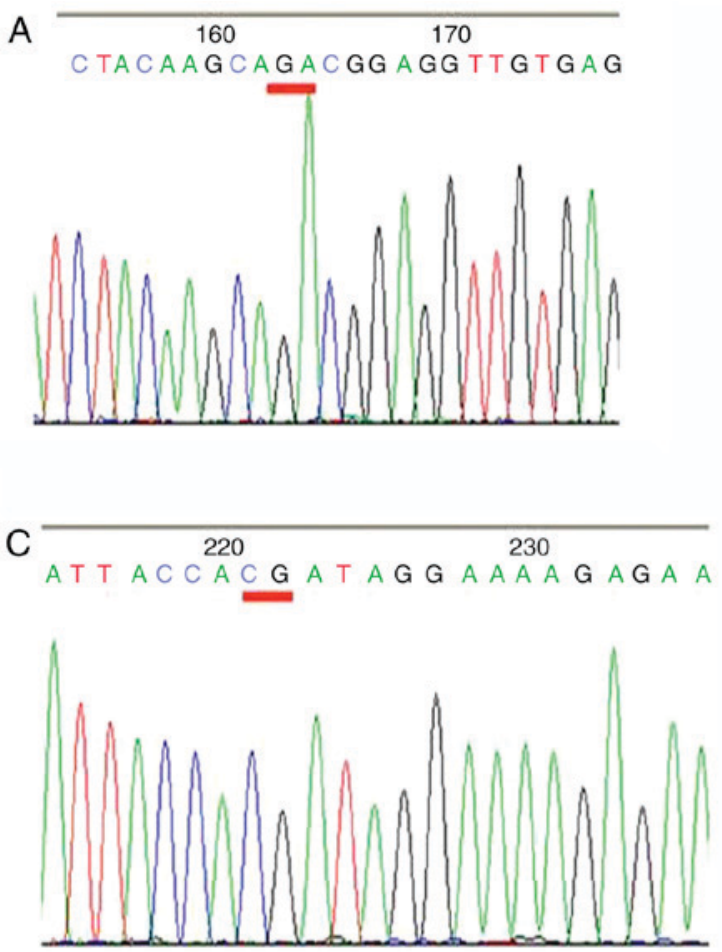
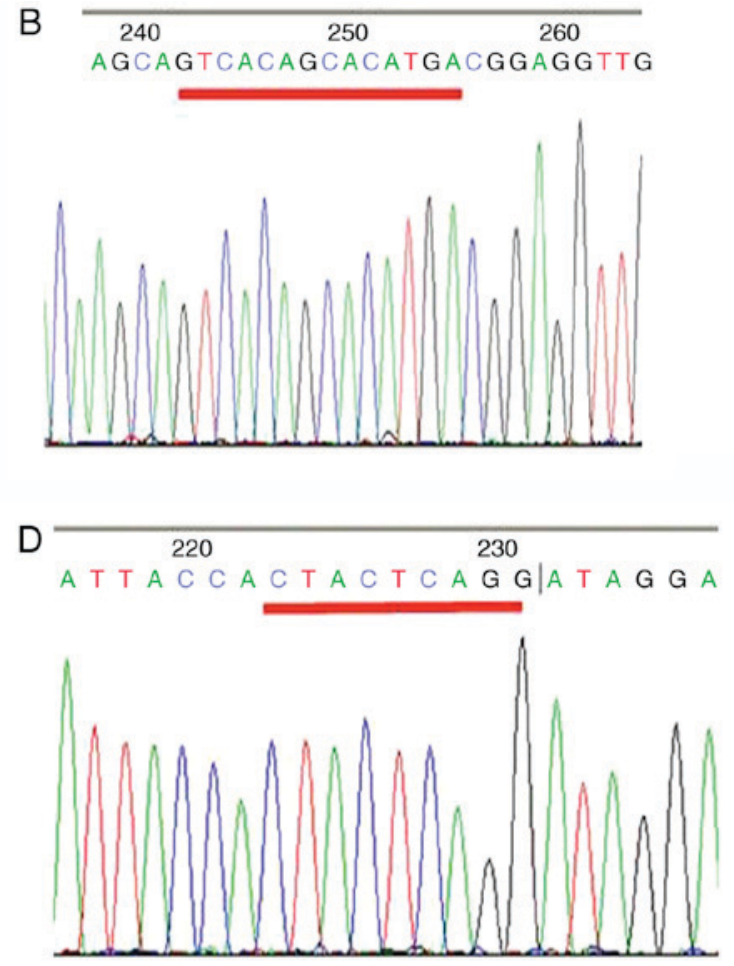

Figure 5. Sanger sequencing chromatograms. (A) TP53-p.V166_H169del and (B) TP53-wild-type; (C) TP53-7577157-63del TACTCAG and (D) TP53-wild-type. TP53, tumor protein 53.

results do not imply that these mutations are associated with $\mathrm{BM}$ in patients with lung cancer as mutations of BRAF (11) and PIK3CA (32) have been identified in a variety of brain tumors and BM; however, to the best of our knowledge, SMAD4 and CTNNB1 mutations have not previously been identified in brain tumors. Mutations of SMAD4 have been identified primarily in pancreatic and colorectal cancer, and among other types of cancer only occasionally ( $\leq 7 \%$ in lung cancer) (33). SMAD4 L533R is a pathogenic mutation, which was first identified in juvenile polyposis (34). Inactivation of SMAD4 was considered to serve an important function in impairing transforming growth factor (TGF)- $\beta$ signaling. Yu et al (35) reported that activation of the TGF- $\beta /$ SMAD signaling pathway promotes LC metastasis by enhancing the ability of LC cells to extravasate into distant organs. It is reasonable to consider that SMAD4 is also involved in LCBM.

Mutational analysis of exon 3 of CTNNB1 in the present study identified p.S45F, p.S37C and p.D32V mutations. These mutations caused amino acid substitutions in 3/15 LCBM samples (Ser33Phe, Ser33Cys and Ser37Cys). The mutations in exon 3 of CTNNB1 affected glycogen synthase kinase-3 $\beta$ phosphorylation sites of the degradation-targeting box of CTNNB1 and resulted in nuclear CTNNB1 protein accumulation (36). Notably, CTNNB1 mutations were detected in $\mathrm{BM}$ from primary LC, in the present study. This finding is consistent with a recent study by Mäki-Nevala et al (37), which demonstrated that CTNNB1 mutations located at codon 37 were detected in two specimens of LCBM. If codons 33 and 37 are a possible location for a number of CTNNB1 mutations in LCBM, this would indicate that CTNNB1 mutations may be important for a secondary event during cancer progression in a carcinogen-specific manner.
There were two novel types of TP53 deletion mutations in LCBM identified in the present study, the TP53 c.495_506del 12 (p.V166_H169del VTAH) and TP53 intron region (7577157-63del TACTCAG), which were validated by Sanger sequencing. Han et al (38) revealed a deletion of two bases, which altered glutamine 167 to alanine, and the resulting frame-shift produced an in-frame stop codon located at amino acid 179 in a human colon cancer cell line (HCC 278). This mutation may be associated with "slippage" or misalignment during DNA replication, due to the occurrence of the CA dinucleotide four times within a stretch of 12 bases (codons 166-169). A novel TP53 gene splice variant was observed in the present study, a 7-base deletion at position 7577157-63 at the splice site of intron 7, which may affect the splice site of exon 8. Although splice sites in TP53 are not typical mutation sites, there is evidence that TP53 splicing mutations lead to exon dropping, indicating a biological relevance (39). Whether these two novel mutations serve a function in BM of lung cancer should be subject to further investigation.

There are several limitations to the present study. The sample size was small, and the retrospective design hindered the ability to study more clinically relevant outcomes, including personalized treatment success, due to the rarity of BM specimens. The NGS approach is designed to detect actionable mutations, including single nucleotide variants, insertions and deletions; however, copy number variants, including gene amplifications and structural variants/rearrangements, are detected less frequently. Therefore, the data acquired may underestimate the mutation burden for the cases. There was no opportunity to investigate matched primary tumors or to analyze in detail intra-patient differences in the mutation status of these genes between tumor sites. 
In conclusion, NGS demonstrated a high-throughput in mutational analysis for the patients with LCBM, calculated by detecting the molecular alterations that have a potential clinical relevance. Such findings may assist in clinical decision-making regarding therapeutic intervention for individual patients and provide improved diagnosis or prognosis.

\section{Acknowledgements}

The present study was supported by the Research Fund for Shanghai Leading Talents (2015-2017).

\section{Competing interests}

The authors declare that they have no competing interests.

\section{References}

1. Mulvenna PM: The management of brain metastases in patients with non-small cell lung cancer is it time to go back to the drawing board? Clin Oncol (R Coll Radiol) 22: 365-373, 2010.

2. Ting CY, Fan CH, Liu HL, Huang CY, Hsieh HY, Yen TC, Wei KC and Yeh CK: Concurrent blood-brain barrier opening and local drug delivery using drug-carrying microbubbles and focused ultrasound for brain glioma treatment. Biomaterials 33 704-712, 2012

3. Cardarella S and Johnson BE: The impact of genomic changes on treatment of lung cancer. Am J Respir Crit Care Med 188: 770-775, 2013.

4. Lindman NI, Cagle PT, Beasley MB, Chitale DA, Dacic S, Giaccone G, Jenkins RB, Kwiatkowski DJ, Saldivar JS, Squire J, et al: Molecular testing guidelines for selection of lung cancer patients for EGFR and ALK tyrosine kinase inhibitors: Guideline from the College of American Pathologists, International Association for the Study of Lung Cancer, and Association for Molecular Pathology. J Thorac 8: 823-859, 2013.

5. Tartarone A, Lazzari C, Lerose R, Conteduca V, Improta G, Zupa A, Bulotta A, Aieta M and Gregorc V: Mechanisms of resistance to EGFR tyrosine kinase inhibitors gefitinib/erlotinib and to ALK inhibitor crizotinib. Lung Cancer 81: 328-336, 2013

6. Lindeman NI, Cagle PT, Beasley MB, Chitale DA, Dacic S, Giaccone G, Jenkins RB, Kwiatkowski DJ, Saldivar JS Squire J, et al: Molecular testing guideline for selection of lung cancer patients for EGFR and ALK tyrosine kinase inhibitors: guideline from the College of American Pathologists, International Association for the Study of Lung Cancer, and Association for Molecular Pathology. Arch Pathol Lab Med 137: 828-860, 2013

7. Shaw AT, Ou SH, Bang YJ, Camidge DR, Solomon BJ, Salgia R, Riely GJ, Varella-Garcia M, Shapiro GI, Costa DB, et al: Crizotinib in ROS1-rearranged non-small-cell lung cancer. N Engl J Med 371: 1963-1971, 2014.

8. Zer A and Leighl N: Promising targets and current clinical trials in metastatic non-squamous NSCLC. Front Oncol 4: 329, 2014.

9. Welsh JW, Komaki R, Amini A, Munsell MF, Unger W, Allen PK, Chang JY, Wefel JS, McGovern SL, Garland LL, et al: Phase II trial of erlotinib plus concurrent whole-brain radiation therapy for patients with brain metastases from non-small-cell lung cancer. J Clin Oncol 31: 895-902, 2013.

10. Gadgeel SM, Gandhi L, Riely GJ, Chiappori AA, West HL, Azada MC, Morcos PN, Lee RM, Garcia L, Yu L, et al: Safety and activity of alectinib against systemic disease and brain metastases in patients with crizotinib-resistant ALK-rearranged non-small-cell lungcancer (AF-002JG): Results from the dose-finding portion of aphase $1 / 2$ study. Lancet Oncol 15 : $1119-1128,2014$

11. Robinson SD, O'Shaughnessy JA, Cowey CL and Konduri K: BRAF V600E-mutated lung adenocarcinoma with metastases to the brain responding to treatment with vemurafenib. Lung Cancer 85: 326-330, 2014.
12. Preusser M, Berghoff AS, Koller R, Zielinski CC, Hainfellner JA, Liebmann-Reindl S, Popitsch N, Geier CB, Streubel B and Birner P: Spectrum of gene mutations detected by next generation exome sequencing in brain metastases of lung adenocarcinoma. Eur J Cancer 51: 1803-1811, 2015.

13. Ellis MJ, Ding L, Shen D, Luo J, Suman VJ, Wallis JW, Van Tine BA, Hoog J, Goiffon RJ, Goldstein TC, et al: Whole-genome analysis informs breast cancer response to aromatase inhibition. Nature 486: 353-360, 2012.

14. Boland GM, Piha-Paul SA, Subbiah V, Routbort M, Herbrich SM, Baggerly K, Patel KP, Brusco L, Horombe C, Naing A, et al: Clinical next generation sequencing to identify actionable aberrations in a phase I program. Oncotarget 6: 20099-20110, 2015.

15. Movva S, Wen W, Chen W, Millis SZ, Gatalica Z, Reddy S, von Mehren M and Van Tine BA: Multi-platform profiling of over 2000 sarcomas: Identification of biomarkers and novel therapeutic targets. Oncotarget 6: 12234-12247, 2015.

16. Sie D, Snijders PJ, Meijer GA, Doeleman MW, van Moorsel MI, van Essen HF, Eijk PP, Grünberg K, van Grieken NC, Thunnissen E, et al: Performance of amplicon-based next generation DNA sequencing for diagnostic gene mutation profiling in oncopathology. Cell Oncol (Dordr) 37: 353-361, 2014.

17. Hagemann IS, Devarakonda S, Lockwood CM, Spencer DH, Guebert K, Bredemeyer AJ, Al-Kateb H, Nguyen TT, Duncavage EJ, Cottrell CE, et al: Clinical next-generation sequencing in patients with non-small cell lung cancer. Cancer 121: 631-639, 2015.

18. Frampton GM, Fichtenholtz A, Otto GA, Wang K, Downing SR, He J, Schnall-Levin M, White J, Sanford EM, An P, et al: Development and validation of a clinical cancer genomic profiling test based on massively parallel DNA sequencing. Nat Biotechnol 31: 1023-1031, 2013.

19. Detterbeck FC, Boffa DJ, Kim AW and Tanoue LT: The 8th edition lung cancer stage classification. CHEST 151: 193-203, 2017.

20. Torre LA, Bray F, Siegel RL, Ferlay J, Lortet-Tieulent J and Jemal A: Global cancer statistics, 2012. CA Cancer J Clin 65: 87-108, 2015.

21. Bailon O, Kallel A, Chouahnia K, Billot S, Ferrari D and Carpentier AF: Management of brain metastases from non-small cell lung carcinoma. Rev Neurol (Paris) 167: 579-591, 2011 (In French).

22. Lemjabbar-Alaoui H, Hassan OU, Yang YW and Buchanan P: Lung cancer: Biology and treatment options. Biochim Biophys Acta 1856: 189-210, 2015.

23. Andrews DW, Scott CB, Sperduto PW, Flanders AE, Gaspar LE, Schell MC, Werner-Wasik M, Demas W, Ryu J, Bahary JP, et al: Whole brain radiation therapy with or without stereotactic radio surgery boost for patients with one to three brain metastases: Phase III results of the RTOG 9508 randomised trial. Lancet 363: 1665-1672, 2004

24. Cancer Genome Atlas Research Network: Comprehensive molecular profiling of lung adenocarcinoma. Nature 511: 543-550, 2014.

25. Imielinski M, Berger AH, Hammerman PS, Hernandez B, Pugh TJ, Hodis E, Cho J, Suh J, Capelletti M, Sivachenko A, et al: Mapping the hallmarks of lung adenocarcinoma with massively parallel sequencing. Cell 150: 1107-1120, 2012.

26. Dienstmann R, Rodon J, Barretina J and Tabernero J: Genomic medicine frontier in human solid tumors: Prospects and challenges. J Clin Oncol 31: 1874-1884, 2013.

27. Russo A, Franchina T, Ricciardi GR, Picone A, Ferraro G, Zanghì M, Toscano G, Giordano A and Adamo V: A decade of EGFR inhibition in EGFR-mutated non small cell lung cancer (NSCLC): Old successes and future perspectives. Oncotarget 6: 26814-26825, 2015.

28. Mao C, Qiu LX, Liao RY, Du FB, Ding H, Yang WC, Li J and Chen Q: KRAS mutations and resistance to EGFR-TKIs treatment in patients with non-small cell lung cancer: A meta-analysis of 22 studies. Lung Cancer 69: 272-278, 2010.

29. Riely GJ, Kris MG, Rosenbaum D, Marks J, Li A, Chitale DA, Nafa K, Riedel ER, Hsu M, Pao W, et al: Frequency and distinctive spectrum of KRAS mutations in never smokers with lung adenocarcinoma. Clin Cancer Res 14: 5731-5734, 2008.

30. Arrieta O, Cardona AF, Martín C, Más-López L, Corrales-Rodríguez L, Bramuglia G, Castillo-Fernandez O, Meyerson M, Amieva-Rivera E, Campos-Parra AD, et al: Updated frequency of EGFR and KRAS mutations in non-small-cell lung cancer in Latin America: The Latin-American Consortium for the Investigation of Lung Cancer (CLICaP). J Thorac Oncol 10: $838-843,2015$. 
31. Zheng D, Wang R, Zhang Y, Pan Y, Cheng X, Cheng C, Zheng S, Li H, Gong R, Li Y, et al: The prevalence and prognostic significance of KRAS mutation subtypes in lung adenocarcinomas from Chinese populations. Onco Targets Ther 9: 833-843, 2016.

32. Paik PK, Shen R, Won H, Rekhtman N, Wang L, Sima CS, Arora A, Seshan V, Ladanyi M, Berger MF and Kris MG: Next generation sequencing of stage IV squamous cell lung cancers reveals an association of PI3K aberrations and evidence of clonal heterogeneity in patients with brain metastases. Cancer Discov 5: 610-621, 2015.

33. Yanagisawa K, Uchida K, Nagatake M, Masuda A, Sugiyama M, Saito T, Yamaki K, Takahashi T and Osada H: Heterogeneities in the biological and biochemical function of Smad2 and Smad4 mutants naturally occurring in human lung cancers. Oncogene 19: 2305-2311, 2000.

34. Gallione CJ, Repetto CJ, Legius E, Rustgi AK, Schelley SL, Tejpar S, Mitchell G, Drouin E, Westermann CJ and Marchuk DA: A combined syndrome of juvenile polyposis and hereditary haemorrhagic telangiectasia associated with mutations in MADH4 (SMAD4). Lancet 363: 852-859, 2004.
35. Yu JR, Tai Y, Jin Y, Hammell MC, Wilkinson JE, Roe JS, Vakoc CR and Van Aelst L: TGF- $\beta$ /Smad signaling through DOCK4 facilitates lung adenocarcinoma metastasis. Genes Dev 29: 250-261, 2015.

36. Kitaewa MN, Grogan L, Williams JP, Dimond E, Nakahara K, Hausner P, DeNobile JW, Soballe PW and Kirsch IR: Mutations in beta-catenin are uncommon in colorectal cancer occurring in occasional replication error-positive tumors. Cancer Res 57: 4478-4481, 1997.

37. Mäki-Nevala S, Sarhadi VK, Rönty M, Kettunen E, Husgafvel-Pursiainen K, Wolff H, Knuuttila A and Knuutila S: Hot spot mutations in Finnish non-small cell lung cancers. Lung Cancer 99: 102-110, 2016

38. Han ES, Moyer MP, Naylor S and Sakaguchi AY: Mutation in the TP53 gene in colorectal carcinoma detected by polymerase chain reaction. Genes Chromosomes Cancer 3: 313-317, 1991.

39. Lai MY, Chang HC, Li HP, Ku CK, Chen PJ, Sheu JC, Huang GT, Lee PH and Chen DS: Splicing mutations of the p53 gene in human hepatocellular carcinoma. Cancer Res 53: 1653-1656, 1993. 